\title{
EXISTENCE OF SOLUTIONS FOR NONLOCAL BOUNDARY VALUE PROBLEM WITH SINGULARITY IN PHASE VARIABLES
}

\author{
Y. TIAN and W. GE \\ Received December 6, 2004
}

\begin{abstract}
In this paper, we prove existence results for singular problem

$$
\left\{x^{(n)}(t)+f\left(t, x(t), \ldots, x^{(n-2)}(t)\right)=0, \quad 0<t<1,\right.
$$$$
\left\{x^{(i)}(0)=0, \quad 0 \leq i \leq n-2, \quad x^{(n-2)}(1)=\int_{0}^{1} x^{(n-2)}(s) d g(s) .\right.
$$

Here the positive Carathédory function $f$ may be singular at the zero value of all its phase variables. Proofs are based on the Leray-Schauder degree and Vitali's convergence theorem.
\end{abstract}

\section{Introduction}

Let $J=[0,1], \mathbb{R}_{-}=(-\infty, 0), \mathbb{R}_{+}=(0, \infty), \mathbb{R}_{0}=\mathbb{R} \backslash\{0\}$.

We investigate the existence of solutions for singular boundary value problem

$$
x^{(n)}(t)+f\left(t, x(t), \ldots, x^{(n-2)}(t)\right)=0, \quad 0<t<1,
$$

2000 Mathematics Subject Classification. 34B16, 34B18.

Key words and phrases. Singular higher-order differential equation, regularization, Vitali's convergence theorem.

Supported by National Natural Sciences Foundation of P. R. China (10371006)

ISSN 1425-6908 C Heldermann Verlag. 


$$
x^{(i)}(0)=0, \quad 0 \leq i \leq n-2, \quad x^{(n-2)}(1)=\int_{0}^{1} x^{(n-2)}(s) d g(s),
$$

where $n \geq 2$, the integral is in the sense of Riemann-Stieltjes and nonlinear term $f$ satisfies local Carathédory conditions on $J \times D(f \in \operatorname{Car}(J \times D))$ with

$$
D=\underbrace{\mathbb{R}_{+} \times \cdots \times \mathbb{R}_{+}}_{n-2} .
$$

The function $f$ in (1.1) may be singular at the zero value of all its phase variables.

Definition 1.1. A function $x \in A C^{n-1}(J)$ (i.e. $x$ has absolutely continuous the $(n-1)^{s t}$ derivative on $\left.J\right)$ is said to be a solution of boundary value problem (1.1)-(1.2), if $x^{(i)}(t)>0$ on $(0,1]$ for $0 \leq i \leq n-2, x$ satisfies the boundary condition (1.2) and (1.1) holds a.e. on $J$.

The purpose of this paper is to give conditions which guarantee the existence of a positive solution to BVP (1.1), (1.2).

This paper is mainly motivated by the works [8]-[9], [13], where the existence of two-point higher order BVPs with singularities in phase variables was studied. In [3], Agarwal et al. consider the existence of solutions for Lidstone boundary value problem as follows

$$
\left\{\begin{array}{l}
(-1)^{n} x^{(2 n)}(t)=f\left(t, x(t), \ldots, x^{(2 n-2)}(t)\right), \quad t \in(0, T), \\
x^{(2 j)}(0)=x^{(2 j)}(T)=0, \quad 0 \leq j \leq n-1,
\end{array}\right.
$$

where $f \in \operatorname{Car}(J \times D)$, and satisfying for a.e. $t \in J$ and for each $\left(x_{0}, \ldots, x_{2 n-2}\right) \in D$,

$$
f\left(t, x_{0}, \ldots, x_{2 n-2}\right) \leq \phi(t)+\sum_{j=0}^{2 n-2} q_{j}(t) \omega_{j}\left(\left|x_{j}\right|\right)+\sum_{j=0}^{2 n-2} h_{j}(t)\left|x_{j}\right|,
$$

where $\phi, h_{j} \in L_{1}(J)$ and $q_{j} \in L_{\infty}(J)$ are nonnegative, $\omega_{j}: \mathbb{R}^{+} \rightarrow \mathbb{R}^{+}$are non-increasing, and

$$
\begin{aligned}
S= & \sum_{i=0}^{n-1} \frac{T^{2(n-i)-3}}{6^{n-i-1}} \int_{0}^{T} t(T-t) h_{2 i}(t) d t \\
& +\sum_{i=0}^{n-2} \frac{T^{2(n-i-2)}}{6^{n-i-2}} \int_{0}^{T} t(T-t) h_{2 i+1}(t) d t<1
\end{aligned}
$$

and

$$
\int_{0}^{T} \omega_{j}(s) d s<\infty, \quad \omega_{j}(u v) \leq \Lambda \omega_{j}(u) \omega_{j}(v),
$$

for $0 \leq j \leq 2 n-2$ and $u, v \in \mathbb{R}_{+}$with a positive constant $\Lambda$. 
Another motivation for this paper is the work [8] and [9], where the nonlocal boundary value problem was considered. But nonlinear term $f$ in all these papers have not singularity. For example, in [9] the existence of a solution of the following boundary value problem

$$
\left\{\begin{array}{l}
x^{(n)}(t)=f\left(t, x(t), x^{\prime}(t), \ldots, x^{(n-1)}(t)\right), \quad t \in(0,1), \\
x^{(i)}(0)=0 \text { for } i=0,1, \ldots, k-1, \\
x^{(j)}(1)=\int_{0}^{1} x^{(j)}(s) d G_{n-j}(s) \text { for } j=k, \ldots, n-1
\end{array}\right.
$$

was studied, where $f:[0,1] \times\left(\mathbb{R}^{m}\right)^{n} \rightarrow \mathbb{R}^{m}$ is a Carathéodory function, $f$ has not singularity in phase variables, $k \in\{1, \ldots, n-1\}$, the function $G_{i}$ $(i=k, \ldots, n-k)$ takes value in linear space of all $m \times m$ square matrices. The method used in [9] is Leray-Schauder degree theory.

Besides, there are many papers studied singular boundary value problems. For example second order singular boundary value problems was investigated in Agarwal [2], Liu Bing [10], Zhang Zhongxin [13] and the references therein. The existence of positive solutions for higher order singular boundary value problem was considered in [1]. Generality speaking, nonlinear term $f\left(t, x_{0}, x_{1}, \ldots, x_{q}\right)$ satisfies the following conditions:

(1) $f\left(t, x_{0}, x_{1}, \ldots, x_{q}\right)$ is non-increasing in $x_{i}$ for each fixed $\left(t, x_{0}, x_{1}, \ldots, x_{i-1}, x_{i+1}, \ldots, x_{q}\right), 0 \leq i \leq q$

(2) $\lim _{x_{i} \rightarrow \infty} f\left(t, x_{0}, x_{1}, \ldots, x_{q}\right)=0$ uniformly on compact subsets of $(0,1) \times(0, \infty)^{n-2}, 1 \leq i \leq n-1$.

By using Leray-Schauder degree theory we get a new result on the existence of solution to boundary value problem (1.1)-(1.2). Meanwhile we remove the restraint (1) and (2) on nonlinear term $f$. The approaches to estimate a priori bound of the solutions to boundary value problem (1.1)(1.2) are different from the corresponding ones of the past work [8,9]. At last we give an example to illustrate our results.

From now on, $\|x\|=\max \{|x(t)|: t \in J\},\|x\|_{L^{1}}=\int_{0}^{1}|x(t)| d t$ and $\|x\|_{\infty}=$ ess $\max \{|x(t)|: 0 \leq t \leq 1\}$ stand for the norm in $C^{0}(J), L_{1}(J)$, and $L_{\infty}(J)$, respectively. For any measurable set $\mathcal{M} \subset \mathbb{R}, \mu(\mathcal{M})$ denotes the Lebesgue measure of $\mathcal{M}$.

The following assumptions imposed upon the function in (1.1) will be used in the paper:

$\left(H_{1}\right) f \in \operatorname{Car}(J \times D)$ and there exists nonnegative functions $\phi, q_{i} \in L_{1}(J)$, $\phi(t) \not \equiv 0, h_{i} \in C(J \times \mathbb{R})$ and non-increasing nonnegative function $\omega_{i} \in L_{1}\left(\mathbb{R}_{+}\right), 0 \leq i \leq n-2$ such that for $(t, x) \in J \times D$,

$$
f\left(t, x_{0}, \ldots, x_{n-2}\right)=\phi(t)+\sum_{i=0}^{n-2} q_{i}(t) \omega_{i}\left(\left|x_{i}\right|\right)+\sum_{i=0}^{n-2} h_{i}\left(t, x_{i}\right)
$$


and $h_{i}$ satisfies

$$
\begin{aligned}
& \lim _{\left|x_{i}\right| \rightarrow \infty} \sup _{t \in[0,1]} \frac{h_{i}\left(t, x_{i}\right)}{\left|x_{i}\right|}=\alpha_{i} \geq 0, \quad \alpha_{i} \text { are any constants in }(0,1), \\
& \quad 0 \leq i \leq n-2, \\
& \omega_{i} \text { satisfies } \\
& \omega_{i}(x y) \leq \Lambda \omega_{i}(x) \omega_{i}(y) \quad \begin{array}{l}
\text { for } x, y \in(0, \infty), \\
\Lambda>0 \text { is a positive constant, }
\end{array} \\
& \int_{0}^{1} \omega_{i}\left(\int_{0}^{t}(t-s)^{n-3-i} s(1-s) d s\right) d t<\infty, 0 \leq i \leq n-3, \\
& \int_{0}^{1} \omega_{n-2}(s(1-s)) d s<\infty ;
\end{aligned}
$$

$\left(H_{2}\right) g$ is Lebesgue measurable, increasing on $J$ and satisfies $g(0)=0$, $g(1)<1$.

The paper is organized as follows. Section 2 presents priori bound of solutions for BVP (1.1)-(1.2). Besides, we prove that some sets of functions containing solutions of our auxiliary regular BVPs are uniformly absolutely continuous on $J$. Section 3 we prove the existence of solution for boundary value problem (1.1)-(1.2). Proof is based on the Arzelà-Ascoli theorem and the Vitali's convergence theorem, see, e.g. [5], [6], [11]. Section 4 present an example to illustrate our main result.

\section{Auxiliary results}

Lemma 2.1. Let $\phi \in L_{1}(J)$ be nonnegative and $\phi(t) \not \equiv 0$. Suppose $x \in$ $A C^{n-1}(J)$ satisfy (1.2) and

$$
\phi(t) \leq-x^{(n)}(t), \quad t \in J .
$$

Then we have on $J$ for $0 \leq i \leq n-1$

$$
\begin{aligned}
x^{(i)}(t) & \geq \frac{\left\|x^{(n-2)}\right\|}{(n-3-i) !} \int_{0}^{t}(t-s)^{n-3-i} s(1-s) d s, \quad 0 \leq i \leq n-3, \\
x^{(n-2)}(t) & \geq\left\|x^{(n-2)}\right\| t(1-t) .
\end{aligned}
$$

Proof. By (1.2) we have

$$
x^{(i)}(t)=\int_{0}^{t} x^{(i+1)}(s) d s, \quad i=0, \ldots, n-3 .
$$


By $(2.1)$, we have $x^{(n-2)}(t)$ is concave on $J$. So

$$
\min _{t \in[0,1]} x^{(n-2)}(t)=\min \left\{x^{(n-2)}(0), x^{(n-2)}(1)\right\} .
$$

We claim $x^{(n-2)}(1) \geq 0$. If not,

$$
\begin{aligned}
x^{(n-2)}(1) & =\int_{0}^{1} x^{(n-2)}(s) d g(s) \geq \min _{t \in[0,1]} x^{(n-2)}(s) \int_{0}^{1} d g(s) \\
& =x^{(n-2)}(1) g(1)>x^{(n-2)}(1),
\end{aligned}
$$

a contradiction. Thus we obtain $x^{(n-2)}(t) \geq 0$ for $t \in J$. So

$$
x^{(n-2)}(t) \geq\left\|x^{(n-2)}\right\| t(1-t) .
$$

By (2.1)-(2.2) we have

$$
x^{(i)}(t) \geq \frac{\left\|x^{(n-2)}\right\|}{(n-3-i) !} \int_{0}^{t}(t-s)^{n-3-i} s(1-s) d s .
$$

Lemma 2.2. Let $\phi \in L_{1}(J)$ be nonnegative and $\phi(t) \not \equiv 0$. Then there exists a positive constant $c=c(\phi)$ such that for each function $x \in A C^{n-1}(J)$ satisfying (1.2) and

$$
\phi(t) \leq-x^{(n)}(t), \quad \text { for a.e. } t \in J
$$

the estimate $\left\|x^{(n-2)}\right\| \geq c$ holds.

Proof. By $-x^{(n)}(t) \geq \phi(t) \geq 0$, we know $x^{(n-2)}(t)$ is concave on $J$. If $x^{(n-2)}(t) \equiv 0, t \in J$, then $x^{(n)}(t) \equiv 0, t \in J$, which contradicts that $-x^{(n)}(t) \geq \phi(t)$ and $\phi(t)$ be nonnegative and $\phi(t) \not \equiv 0$.

Remark 2.1. It follows from Lemma 2.1 and Lemma 2.2 that for any solution of BVP (1.1)-(1.2)

$$
\begin{aligned}
\left|x^{(i)}(t)\right| & \geq \frac{c}{(n-3-i) !} \int_{0}^{t}(t-s)^{n-3-i} s(1-s) d s, \quad i=0, \ldots, n-3, \\
x^{(n-2)} & \geq c t(1-t),
\end{aligned}
$$

where $c=c(\phi)$.

For each $m \in \mathbb{N}$, define $\mathcal{X}_{m}$, and $f_{m} \in \operatorname{Car}\left(J \times \mathbb{R}^{n}\right)$ by the formulas

$$
\mathcal{X}_{m}(u)= \begin{cases}u, & \text { for } u \geq \frac{1}{m} \\ \frac{1}{m}, & \text { for } u<\frac{1}{m}\end{cases}
$$


and

$$
\begin{aligned}
f_{m}\left(t, x_{0}, x_{1}, \ldots, x_{n-2}\right)= & \phi(t)+\sum_{i=0}^{n-2} q_{i}(t) \omega_{i}\left(\mathcal{X}_{m}\left(x_{i}\right)\right) \\
& +\sum_{i=0}^{n-2} h_{i}\left(t, x_{i}\right)
\end{aligned}
$$

for $\left(t, x_{0}, \ldots, x_{n-2}\right) \in J \times \mathbb{R}^{n-1}$. Hence

$$
\begin{aligned}
0<\phi(t) & \leq f_{m}\left(t, x_{0}, \ldots, x_{n-2}\right) \\
& \leq \phi(t)+\sum_{i=0}^{n-2} q_{i}(t) \omega_{i}\left(\left|x_{i}\right|\right)+\sum_{i=0}^{n-2} h_{i}\left(t, x_{i}\right)
\end{aligned}
$$

for a.e. $t \in J$ and each $\left(x_{0}, \ldots, x_{n-2}\right) \in \mathbb{R}_{0}^{n-1}$.

Consider auxiliary regular differential equation

$$
x^{(n)}(t)+f_{m}\left(t, x(t), \ldots, x^{(n-2)}(t)\right)=0
$$

and

$$
x^{(n)}(t)+\lambda f_{m}\left(t, x(t), \ldots, x^{(n-2)}(t)\right)=0, \quad \lambda \in[0,1]
$$

depending on the parameters $m \in \mathbb{N}$.

Lemma 2.3. Let $h:[0,1] \rightarrow \mathbb{R}_{+}$be continuous. Suppose $x(t)$ is a solution of the following boundary value problem

$$
\left\{\begin{array}{l}
x^{(n)}(t)+h(t)=0, \quad t \in(0,1), \\
x^{(i)}(0)=0, \quad i=0, \ldots, n-2, \quad x^{(n-2)}(1)=\int_{0}^{1} x^{(n-2)}(s) d g(s) .
\end{array}\right.
$$

Then $x(t)$ can be uniquely expressed as

$$
x(t)=\frac{A t^{n-1}}{(n-1) !}-\int_{0}^{t} \frac{(t-s)^{n-1}}{(n-1) !} h(s) d s
$$

where

$$
A=\frac{1}{1-\int_{0}^{1} s d g(s)}\left(\int_{0}^{1}(1-s) h(s) d s-\int_{0}^{1}\left(\int_{0}^{r}(r-s) h(s) d s\right) d g(r)\right) .
$$

Proof. Sufficiency. First integrating both sides of equation $x^{(n)}(t)+h(t)=$ 0 on $[0, t]$, we have

$$
x^{(n-1)}(t)=x^{(n-1)}(0)-\int_{0}^{t} h(s) d s .
$$


Integrating again the above equation on $[0, t]$ and using the second boundary condition we get

$$
x^{(n-2)}(t)=x^{(n-1)}(0) t-\int_{0}^{t}(t-s) h(s) d s .
$$

It follows that

$$
\int_{0}^{1} x^{(n-2)}(s) d g(s)=x^{(n-1)}(0) \int_{0}^{1} s d g(s)-\int_{0}^{1}\left(\int_{0}^{r}(r-s) h(s) d s\right) d g(r) .
$$

Noticing boundary condition

$$
x^{(n-2)}(1)=\int_{0}^{1} x^{(n-2)}(s) d g(s),
$$

we obtain the following equality

$$
\begin{aligned}
x^{(n-1)}(0)-\int_{0}^{1}(1-s) h(s) d s= & x^{(n-1)}(0) \int_{0}^{1} s d g(s) \\
& -\int_{0}^{1}\left(\int_{0}^{r}(r-s) h(s) d s\right) d g(r),
\end{aligned}
$$

holds, which means

$$
\begin{aligned}
x^{(n-1)}(0) & =\frac{1}{1-\int_{0}^{1} s d g(s)}\left(\int_{0}^{1}(1-s) h(s) d s-\int_{0}^{1}\left(\int_{0}^{r}(r-s) h(s) d s\right) d g(r)\right) \\
& =A .
\end{aligned}
$$

So

$$
x^{(n-2)}(t)=A t-\int_{0}^{t}(t-s) h(s) d s .
$$

Integrating the above equation on $[0, t]$ for $n-2$ times, we get

$$
x(t)=\frac{A t^{n-1}}{(n-1) !}-\int_{0}^{t} \frac{(t-s)^{n-1}}{(n-1) !} h(s) d s
$$

holds.

Necessity. From the expression of $x$, it is easy to obtain $x$ is a solution of boundary value problem in Lemma 2.3.

Lemma 2.4. Let $m \in \mathbb{N}$. If there exists a positive constant $K$ such that

$$
\left\|x^{(j)}\right\| \leq K, \quad 0 \leq j \leq n-1
$$

for any solution $x$ of $B V P(2.7)$, (1.2) with $\lambda \in[0,1]$, then $B V P(2.6),(1.2)$ has a solution $x$ satisfying (2.8). 
Proof. By Lemma 2.3 we know that solving (2.7), (1.2) is equivalent to find $x \in C^{n-1}(J)$ satisfying

$$
x(t)=\lambda \frac{A t^{n-1}}{(n-1) !}-\lambda \int_{0}^{t} \frac{(t-s)^{n-1}}{(n-1) !} f_{m}\left(s, x(s), \ldots, x^{(n-1)}(s)\right) d s,
$$

where $A$ is defined in Lemma 2.3. It is easy to see that

$$
\begin{aligned}
& S: C^{n-1}(J) \rightarrow C^{n-1}(J), \\
& (S x)(t)=\frac{A t^{n-1}}{(n-1) !}-\int_{0}^{t} \frac{(t-s)^{n-1}}{(n-1) !} f_{m}\left(s, x(s), \ldots, x^{(n-1)}(s)\right) d s
\end{aligned}
$$

is a completely continuous operator. Since we can rewrite (2.9) as

$$
x=\lambda S x, \quad \lambda \in[0,1] .
$$

By our assumption, (2.10) holds for any solution of (2.7), there exists a solution $x$ of the operator equation $x=S x$ by [6]. Of course, $x$ is a solution of BVP (2.6), (1.2) satisfying (2.8).

For convenience we denote

$$
\begin{aligned}
\Gamma:= & \int_{0}^{1}\left(\phi(s)+\Lambda \sum_{i=0}^{n-3} q_{i}(s) \omega_{i}\left(\frac{c}{(n-3-i) !}\right) \omega_{i}\left(\int_{0}^{s}(s-\theta)^{n-3-i} \theta(1-\theta) d \theta\right)\right. \\
& \left.+q_{n-2}(s) \Lambda \omega_{n-2}(c) \omega_{n-2}(s(1-s))\right) d s .
\end{aligned}
$$

Lemma 2.5. Let assumptions $\left(H_{1}\right)-\left(H_{2}\right)$ be satisfied. Furthermore, the following inequality is satisfied

$$
\sum_{i=0}^{n-2} \frac{\alpha_{i}}{(n-i-1) !}<1 .
$$

Then there exists a positive constant $P$ such that $\left\|x^{(j)}\right\| \leq P, 0 \leq j \leq n-1$ for any solution $x$ of $B V P(2.7)$, (1.2) with $m \in \mathbb{N}$.

Proof. Let $x$ be a solution of BVP (2.7), (1.2) for some $m \in \mathbb{N}$.

In what follows we will prove $\left\|x^{(i)}\right\| \leq P, 0 \leq j \leq n-1$. The proof of this lemma is divided into three steps.

Step 1. It follows from boundary condition that

$$
x^{(i)}(t)=\int_{0}^{t} \frac{(t-\theta)^{n-i-2}}{(n-i-2) !} x^{(n-1)}(\theta) d \theta, \quad t \in J, \quad 0 \leq i \leq n-2 .
$$

Thus we have

$$
\left\|x^{(i)}\right\| \leq \frac{1}{(n-i-1) !}\left\|x^{(n-1)}\right\|, \quad 0 \leq i \leq n-2 .
$$


Step 2. Prove there exists a positive constant $P$ such that

$$
\left\|x^{(n-1)}\right\| \leq P .
$$

We claim there exists $\xi \in[0,1]$ such that $x^{(n-1)}(\xi)=0$.

Otherwise, if $x^{(n-1)}(t) \geq 0, t \in[0,1]$, then

$$
x^{(n-2)}(1)=\max _{t \in J} x^{(n-2)}(t) .
$$

But

$$
x^{(n-2)}(1)=\int_{0}^{1} x^{(n-2)}(s) d g(s) \leq \max _{t \in J} x^{(n-2)}(t) g(1)<x^{(n-2)}(1),
$$

a contradiction;

if $x^{(n-1)}(t) \leq 0, t \in[0,1]$, then $x^{(n-2)}(t) \leq 0$ for $t \in J$. But

$$
x^{(n-2)}(1)=\int_{0}^{1} x^{(n-2)}(s) d g(s) \geq \min _{t \in J} x^{(n-2)}(t) g(1)>x^{(n-2)}(1),
$$

a contradiction.

Noticing $x^{(n-1)}(t)$ is decreasing on $[0,1]$, one has

$$
x^{(n-1)}(t)>0 \text { for } t \in[0, \xi), \quad x^{(n-1)}(t)<0 \text { for } t \in(\xi, 1] .
$$

Let sufficiently small $\varepsilon>0$ be such that

$$
\sum_{i=0}^{n-2} \frac{\alpha_{i}+\varepsilon}{(n-i-1) !}<1 .
$$

Then for this $\varepsilon>0$, there is $\delta>0$ so that

$$
\begin{aligned}
\left|h_{i}\left(t, x_{i}\right)\right|<\left(\alpha_{i}+\varepsilon\right)\left|x_{i}\right| & \text { uniformly for } t \in[0,1], \\
& \text { and }\left|x_{i}\right|>\delta, i=0, \ldots, n-2 .
\end{aligned}
$$

Let, for $i=0, \ldots, n-2$,

$$
\begin{gathered}
\Delta_{1, i}=\left\{t: t \in[0,1],\left|x_{i}(t)\right| \leq \delta\right\}, \\
\Delta_{2, i}=\left\{t: t \in[0,1],\left|x_{i}(t)\right|>\delta\right\}, \\
h_{\delta, i}=\max _{t \in[0,1],\left|x_{i}\right| \leq \delta} h_{i}\left(t, x_{i}\right) .
\end{gathered}
$$

On the one hand, integrating both sides of (2.7) from $t$ to $\xi,(t \in[0, \xi])$, using (2.5), Remark 2.1, (2.11) and (2.15) we have

$$
\begin{aligned}
x^{(n-1)}(t) & =\lambda \int_{t}^{\xi} f_{m}\left(s, x(s), \ldots, x^{(n-2)}(s)\right) d s \\
& \leq \lambda \int_{t}^{\xi}\left(\phi(s)+\sum_{i=0}^{n-2} q_{i}(s) \omega_{i}\left(\left|x^{(i)}(s)\right|\right)\right) d s
\end{aligned}
$$




$$
\begin{aligned}
& +\sum_{i=0}^{n-2} \int_{[t, \xi] \cap \Delta_{1, i}} h_{i}\left(s, x^{(i)}(s)\right) d s+\sum_{i=0}^{n-2} \int_{[t, \xi] \cap \Delta_{2, i}} h_{i}\left(s, x^{(i)}(s)\right) d s \\
\leq & \int_{t}^{\xi}\left[\phi(s)+\sum_{i=0}^{n-3} q_{i}(s) \Lambda \omega_{i}\left(\frac{c}{(n-3-i) !}\right)\right. \\
& \times \omega_{i}\left(\int_{0}^{s}(s-\theta)^{n-3-i} \theta(1-\theta) d \theta\right) \\
& \left.+q_{n-2}(s) \Lambda \omega_{n-2}(c) \omega_{n-2}(s(1-s))\right] \\
& +\sum_{i=0}^{n-2} \int_{[t, \xi] \cap \Delta_{1, i}} h_{i}\left(s, x^{(i)}(s)\right) d s+\sum_{i=0}^{n-2} \int_{[t, \xi] \cap \Delta_{2, i}} h_{i}\left(s, x^{(i)}(s)\right) d s
\end{aligned}
$$

thus we have for $t \in[0, \xi]$, noticing (2.12)

$$
\begin{aligned}
x^{(n-1)}(t) & \leq \Gamma+\sum_{i=0}^{n-2} h_{\delta, i}+\int_{t}^{\xi} \sum_{i=0}^{n-2}\left(\alpha_{i}+\varepsilon\right)\left|x^{(i)}(s)\right| d s \\
& \leq \Gamma+\sum_{i=0}^{n-2} h_{\delta, i}+\sum_{i=0}^{n-2} \frac{\alpha_{i}+\varepsilon}{(n-i-1) !}\left\|x^{(n-1)}\right\|,
\end{aligned}
$$

i.e.

$$
x^{(n-1)}(0) \leq \Gamma+\sum_{i=0}^{n-2} h_{\delta, i}+\sum_{i=0}^{n-2} \frac{\alpha_{i}+\varepsilon}{(n-i-1) !}\left\|x^{(n-1)}\right\| .
$$

On the other hand, integrating both sides of (2.7) from $\xi$ to $t,(t \in[\xi, 1])$, using (2.5), Remark $2.1,(2.11),(2.15)$, we have

$$
\begin{aligned}
\left|x^{(n-1)}(t)\right|= & \lambda \int_{\xi}^{t} f_{m}\left(s, x(s), \ldots, x^{(n-2)}(s)\right) d s \\
\leq & \lambda \int_{\xi}^{t}\left(\phi(s)+\sum_{i=0}^{n-2} q_{i}(s) \omega_{i}\left(\left|x^{(i)}(s)\right|\right)\right) d s \\
& +\sum_{i=0}^{n-2} \int_{[\xi, t] \cap \Delta_{1, i}} h_{i}\left(s,\left|x^{(i)}(s)\right|\right)+\sum_{i=0}^{n-2} \int_{[\xi, t] \cap \Delta_{2, i}} h_{i}\left(s,\left|x^{(i)}(s)\right|\right) d s \\
\leq & \lambda \int_{\xi}^{t}\left[\phi(s)+\sum_{i=0}^{n-2} q_{i}(s) \Lambda \omega_{i}\left(\frac{c}{(n-3-i) !}\right)\right. \\
& \times \omega_{i}\left(\int_{0}^{s}(s-\theta)^{n-3-i} \theta(1-\theta) d \theta\right)
\end{aligned}
$$


EXISTENCE OF SOLUTIONS FOR NONLOCAL BOUNDARY VALUE PROBLEM 103

$$
\begin{aligned}
& \left.+q_{n-2}(s) \Lambda \omega_{n-2}(c) \omega_{n-2}(s(1-s))\right] d s \\
& +\sum_{i=0}^{n-2} \int_{[\xi, t] \cap \Delta_{1, i}} h_{i}\left(s, x^{(i)}(s)\right)+\sum_{i=0}^{n-2} \int_{[\xi, t] \cap \Delta_{2, i}} h_{i}\left(s, x^{(i)}(s)\right) d s,
\end{aligned}
$$

thus for $t \in[\xi, 1]$, noticing (2.12) we have

$$
\begin{aligned}
\left|x^{(n-1)}(s)\right| & \leq \Gamma+\sum_{i=0}^{n-2} h_{\delta, i}+\sum_{i=0}^{n-2} \int_{\xi}^{t}\left(\alpha_{i}+\varepsilon\right)\left|x^{(i)}(s)\right| d s \\
& \leq \Gamma+\sum_{i=0}^{n-2} h_{\delta, i}+\sum_{i=0}^{n-2} \frac{\alpha_{i}+\varepsilon}{(n-i-1) !}\left\|x^{(n-1)}\right\|,
\end{aligned}
$$

i.e.

$$
\left|x^{(n-1}(1)\right| \leq \Gamma+\sum_{i=0}^{n-2} h_{\delta, i}+\sum_{i=0}^{n-2} \frac{\alpha_{i}+\varepsilon}{(n-i-1) !}\left\|x^{(n-1)}\right\| .
$$

By $x^{(n-1)}$ is decreasing on $J$, it follows from (2.16) (2.18) that

$$
\left\|x^{(n-1)}\right\|=\max \left\{x^{(n-1)}(0),\left|x^{(n-1)}\right|\right\} \leq \Gamma+\sum_{i=0}^{n-2} h_{\delta, i}+\sum_{i=0}^{n-2} \frac{\alpha_{i}+\varepsilon}{(n-i-1) !}\left\|x^{(n-1)}\right\|
$$

we have

$$
\left\|x^{(n-1)}\right\| \leq \frac{\Gamma+\sum_{i=0}^{n-2} h_{\delta, i}}{1-\sum_{i=0}^{n-2} \frac{\alpha_{i}+\varepsilon}{(n-i-1) !}}:=P .
$$

By $\left(H_{1}\right),\left(H_{3}\right)$ we have $P<\infty$.

Step 3. Prove $\left\|x^{(i)}\right\| \leq P$ for $i=0,1, \ldots, n-1$.

By Step 1 the result is clear. This completes the proof.

Lemma 2.6. Let assumptions $\left(H_{1}\right),\left(H_{3}\right)$ be satisfied. Let $\left\{x_{m}\right\}$ be a sequence of solutions to $B V P(2.7)$, (1.2) for each $m \in \mathbb{N}$. Then the sequence

$$
\left\{f_{m}\left(t, x_{m}(t), \ldots, x_{m}^{(n-2)}(t)\right)\right\} \subset L_{1}(J)
$$

is uniformly absolutely continuous on $J$, that is for each $\varepsilon>0$ there exists $\delta>0$ such that

$$
\int_{\mathcal{M}} f_{m}\left(t, x_{m}(t), \ldots, x_{m}^{(n-2)}(t)\right) d t<\varepsilon
$$

for any measurable set $\mathcal{M} \subset J, \mu(\mathcal{M})<\delta$. 
Proof. With respect to (2.5) and properties of measurable sets, it is sufficient to verify that for every $\varepsilon>0$, there exists $\delta>0$ such that for any at most countable set $\left\{\left(a_{j}, b_{j}\right)\right\}_{j \in J}$ of mutually disjoint intervals $\left\{\left(a_{j}, b_{j}\right)\right\}_{j \in J}$ with $\sum_{j \in J}\left(b_{j}-a_{j}\right)<\delta$, we have for each $m \in \mathbb{N}$,

$$
\sum_{j \in J} \int_{a_{j}}^{b_{j}}\left[\phi(t)+\sum_{i=0}^{n-2} q_{i}(t) \omega_{i}\left(\left|x_{m}^{(i)}\right|\right)+\sum_{i=0}^{n-2} h_{i}\left(t, x_{m}^{(i)}\right)\right] d t<\varepsilon .
$$

By (2.5) we have

$$
\phi(t) \leq f_{m}\left(t, x(t), \ldots, x^{(n-2)}(t)\right), \quad t \in J
$$

Thus the conditions in Lemma 2.1 and Lemma 2.2 are satisfied. There exists $c=c(\phi)$ such that

$$
\begin{aligned}
x_{m}^{(i)}(t) \geq \frac{c}{(n-i-3) !} \int_{0}^{t}(t-s)^{n-3-i} s(1-s) d s, \\
i=0, \ldots, n-3, \quad t \in J, \\
x_{m}^{(n-2)}(t) \geq c t(1-t), \quad t \in[0,1] .
\end{aligned}
$$

In addition by Lemma 2.5 one has

$$
\left\|x_{m}^{(i)}\right\| \leq P, \quad i=0, \ldots, n-2 .
$$

It follows from $(2.20)-(2.22)$ that

$$
\begin{aligned}
& \sum_{j \in J} \int_{a_{j}}^{b_{j}}\left[\phi(t)+\sum_{i=0}^{n-2} q_{i}(t) \omega_{i}\left(\left|x_{m}^{(i)}\right|\right)+\sum_{i=0}^{n-2} h_{i}\left(t, x_{m}^{(i)}\right)\right] d t \\
& \leq \sum_{j \in J} \int_{a_{j}}^{b_{j}}\left[\phi(t)+\Lambda \sum_{i=0}^{n-3} q_{i}(t) \omega_{i}\left(\frac{c}{(n-3-i) !}\right) \omega_{i}\left(\int_{0}^{t}(t-s)^{n-3-i} s(1-s) d s\right)\right. \\
& \left.+\Lambda \sum_{i=0}^{n-2} q_{i}(t) \omega_{i}(c) \omega_{i}(t(1-t))+\sum_{i=0}^{n-2} \max _{\left(t, x_{i}\right) \in[0,1] \times[0, P]} h_{i}\left(t, x_{i}\right)\right] d t .
\end{aligned}
$$

By $\left(H_{1}\right)$, we have $\phi, q_{i}, h_{i}, \in L_{1}(J)$ and (1.6) hold. Consequently, for each $\varepsilon>0$ there exists $\delta>0$ such that for any at most countable set $\left\{\left(a_{j}, b_{j}\right)\right\}_{j \in J}$ of mutually disjoint intervals $\left(a_{j}, b_{j}\right) \subset J$ with $\sum_{j \in J}\left(b_{j}-a_{j}\right)<\delta$, (2.19) holds. This completes the proof.

\section{Existence results}

Theorem 3.1. Suppose the assumptions $\left(H_{1}\right),\left(H_{2}\right)$ and $\left(H_{3}\right)$ be satisfied. Then there exists a solution $x$ such that $x \in A C^{(n-1)}(J), x^{(i)}(t) \geq 0, t \in J$ for $B V P$ (1.1), (1.2). 
Proof. For each $m \in \mathbb{N}$, there exists a solution $x_{m}$ of BVP (2.6), (1.2) by Lemma 2.4, Lemma 2.5. Consider the solution sequence $\left\{x_{m}\right\}$. Lemma 2.5 shows that $\left\{x_{m}\right\}$ is bounded in $C^{n-1}(J)$. Lemma 2.1 and Lemma 2.2 means that

$$
\begin{aligned}
x_{m}^{(i)}(t) & \geq c \int_{0}^{t}(t-s)^{n-3-i} s(1-s) d s, \quad 0 \leq i \leq n-3, \\
x_{m}^{(n-2)}(t) & \geq c t(1-t), \quad t \in J .
\end{aligned}
$$

The Arzelà-Ascoli theorem guarantees the existence of a subsequence $\left\{x_{m_{k}}\right\}$ converging in $C^{n-2}(J)$. Lemma 2.2 means that $c$ is independent $m$, so $(3.1)$ and (3.2) gives

$$
\begin{aligned}
x^{(i)}(t) & \geq c \int_{0}^{t}(t-s)^{n-3-i} s(1-s) d s, \quad i=0, \ldots, n-3, \\
x^{(n-2)}(t) & \geq c t(1-t), \quad t \in J .
\end{aligned}
$$

Moreover,

$$
\begin{aligned}
\lim _{m \rightarrow \infty} x_{m}^{(i)}(0) & =x^{(i)}(0)=0, \quad i=0, \ldots, n-2, \\
\lim _{m \rightarrow \infty} x_{m}^{(n-2)}(1) & =x^{(n-2)}(1) .
\end{aligned}
$$

By Riemann-Stieltjes dominated convergence theorem we have

$$
\lim _{m \rightarrow \infty} \int_{0}^{1} x_{m}^{(n-2)}(s) d g(s)=\int_{0}^{1} x^{(n-2)}(s) d g(s) .
$$

(3.3), (3.4) means $x$ satisfies the boundary condition (1.2).

From $f_{m_{k}} \in \operatorname{Car}\left(J \times \mathbb{R}^{n}\right)$, and their construction, it follows that there exists $\mathcal{M} \in J, \mu(\mathcal{M})=0$ such that $f_{m_{k}}(t, \cdot, \ldots, \cdot)$ are continuous on $\mathbb{R}^{n-1}$ for each $t \in J \backslash \mathcal{M}$, which implies that

$$
\lim _{k \rightarrow \infty} f_{m_{k}}\left(t, x_{m_{k}}(t), \ldots, x_{m_{k}}^{(n-2)}(t)\right)=f\left(t, x(t), \ldots, x^{(n-2)}(t)\right)
$$

for $t \in J \backslash \mathcal{M} \cup\{0\}$.

By Lemma $2.6\left\{f_{m_{k}}\left(t, x_{m_{k}}(t), \ldots, x_{m_{k}}^{(n-2)}(t)\right)\right\}$ is uniformly absolutely continuous on $J$. Hence $f \in L_{1}(J)$ and

$$
\lim _{k \rightarrow \infty} \int_{0}^{t} f_{m_{k}}\left(s, x_{m_{k}}(s), \ldots, x_{m_{k}}^{(n-2)}(s)\right) d s=\int_{0}^{t} f\left(s, x(s), \ldots, x^{(n-2)}(s)\right) d s
$$

for $t \in J$ by the Vitali's convergence theorem. Since $\left\{x_{m_{k}}^{(n-1)}\right\}$ is bounded, we can assume that it is convergent, say

$$
\lim _{k \rightarrow \infty} x_{m_{k}}^{(n-1)}(0)=C .
$$


Taking limits as $k \rightarrow \infty$ in the following equalities

$$
x_{m_{k}}^{(n-2)}(t)=x_{m_{k}}^{(n-1)}(0) t-\int_{0}^{t} \int_{0}^{s} f_{m_{k}}\left(r, x_{m_{k}}(r), \ldots, x_{m_{k}}^{(n-2)}(r)\right) d r d s, \quad t \in J
$$

we get

$$
x^{(n-2)}(t)=C t-\int_{0}^{t} \int_{0}^{s} f\left(r, x(r), \ldots, x^{(n-2)}(r)\right) d r d s, \quad t \in J .
$$

Then $x \in A C^{n-1}(J)$ and

$$
x^{(n)}(t)+f\left(t, x(t), \ldots, x^{(n-1)}(t)\right)=0, \quad \text { for a.e. } t \in J .
$$

Therefore, $x$ is a solution of BVP (1.1), (1.2).

\section{Example}

Let us consider the following third-order boundary value problem

$$
\left\{\begin{array}{l}
x^{(3)}+\phi(t)+\frac{q_{0}(t)}{x_{0}^{1 / 4}}+\frac{q_{1}(t)}{x_{1}^{1 / 2}}+a_{0}(t) \sin \left(\left|x_{0}\right|\right)+a_{1}(t)\left|x_{1}\right|=0, \\
(0)=x^{\prime}(0)=0, \quad x^{\prime}(1)=\int_{0}^{1} x^{\prime}(s) d g(s)
\end{array}\right.
$$

with $\phi, q_{i} \in L_{1}(J), a_{i} \in C(J)$ be positive for $i=0,1, g$ is Lebesgue measurable, increasing on $J$ and satisfies $g(0)=0, g(1)<1$. Corresponding to BVP (1.1)-(1.2), we have

$$
\begin{aligned}
& f\left(t, x_{0}, x_{1}\right)=\phi(t)+\frac{q_{0}(t)}{x_{0}^{1 / 4}}+\frac{q_{1}(t)}{x_{1}^{1 / 2}}+a_{0}(t) \sin \left(\left|x_{0}\right|\right)+a_{1}(t)\left|x_{1}\right|, \\
& \omega_{0}\left(\left|x_{0}\right|\right)=\frac{1}{x_{0}^{1 / 4}}, \quad \omega_{1}\left(x_{1}\right)=\frac{1}{x_{0}^{1 / 2}}, \\
& h_{0}\left(t, x_{0}\right)=a_{0}(t) \sin \left(\left|x_{0}\right|\right), \quad h_{1}\left(t, x_{1}\right)=a_{1}(t)\left|x_{1}\right| .
\end{aligned}
$$

Assume

$$
\sup _{t \in[0,1]} a_{1}(t)<1
$$

holds. Then (4.1) has at least one positive solution $x \in C^{2}(J), x^{\prime \prime} \in A C(J)$.

To see that (4.1) has a positive solution $x \in C^{2}(J), x^{\prime \prime} \in A C(J)$, we notice

$$
\begin{aligned}
\int_{0}^{1} \omega_{0}\left(\int_{0}^{t} s(1-s) d s\right) d t & =\sqrt[4]{6} \int_{0}^{1} \frac{1}{\sqrt[4]{3 t^{2}-2 t^{3}}}<\sqrt[4]{6} \int_{0}^{1} \frac{1}{\sqrt{t}} d t=2 \sqrt[4]{6}<\infty \\
\int_{0}^{1} \omega_{1}(s(1-s)) d s & =\int_{0}^{1} \frac{1}{\sqrt{s(1-s)}} d s<\infty
\end{aligned}
$$


so $\left(H_{1}\right)$ is satisfied. $\left(H_{2}\right)$ is clear to be satisfied. It is easy to see $\left(H_{3}\right)$ are true since (4.2) hold. Theorem 3.1 now guarantees that BVP (4.1) has a solution $x \in A C^{n-1}(J), x^{(i)}(t) \geq 0, i=0,1$.

\section{References}

[1] Agarwal, R. P., O'Regan, D., Wong, P. J. Y., Positive Solutions of Differential, Difference and Integral Equations, Kluwer Academic Publishers, Dordrecht, 1999.

[2] Agarwal, R. P., O'Regan, D., Singular Differential and Integral Equations with Applications, Kluwer Academic Publishers, Dordrecht, 2003.

[3] Agarwal, R. P., O’Regan, D., Rachunková, I., Staněk, S., Two-point higher-order BVPs with singularities in phase variables, Comput. Math. Appl. 46 (2003) 17991826.

[4] Agarwal, R. P., O'Regan, D., Staněk, S., Singular lidstone boundary value problem with given maximal values for solutions, Nonlinear Anal. 55 (2003), 859-881.

[5] Bartle, R. G., A Morden Theory of Integration, Grad. Stud. Math. 32, Amer. Math. Soc., Providence, RI, 2001.

[6] Deimling, K., Nonlinear Functional Analysis, Springer, Berlin-Heidelberg, 1985.

[7] Eloe, P. W., Henderson, J., Positive solutions for $(n-1,1)$ conjugate boundary value problems, Nonlinear Anal. 28 (1997), 1669-1680.

[8] Karakostas, G. L., Tsamatos, P. Ch., On a nonlocal boundary value problem at resonance, J. Math. Anal. Appl. 259 (2001), 209-218.

[9] Karakostas, G. L., Tsamatos, P. Ch., Nonlocal boundary vector value problems for ordinary differential equations of higher order, Nonlinear Anal. 51 (2002), 1421-1427.

[10] Liu, Bing, Positive solutions of three-point boundary value problems for the onedimensional p-Laplacian with infinitely many singularities, Appl. Math. Lett. 17 (2002), 655-661.

[11] Natanson, I. P., Theorie der Funktionen einer reelen Veränderlichen, AkademieVerlag, Berlin, 1969.

[12] Rachunková, I., Staněk, S., A singular boundary value problem for odd-order differential equations, J. Math. Anal. Appl. 291 (2004), 741-756.

[13] Zhang, Zhongxin, Wang, Junyu, The upper and lower solution method for a class of singular nonlinear second order three-point boundary value problems, J. Comput. Appl. Math. 147 (2002), 41-52.

YU TIAN

Department of APPLiED

MATHEMATICS

Weigao Ge

Beijing Institute of Technology

BEIJING 100081

Department of ApPlied

MATHEMATICS

P. R. China

BEIJING 100081

E-MAIL: TIANYU2992@163.COM

Beijing Institute of Technology

P. R. CHINA 